\title{
CLOSTRIDIUM DIFFICILE INFECTION IN CHILDREN: EXPERIENCE IN ATERCIARY CARE HOSPITAL
}

A. Calvo Cillán ${ }^{1}$, L. Martin Pedraz ${ }^{1}$, B. Carazo Gallego ${ }^{2}$, D. Lopez Martin ${ }^{1}$, C. Sole Mesalles ${ }^{3}$, B. Palop Borras 4 , D. Moreno Perez ${ }^{2}$

${ }^{1}$ Hospital Materno Infantil de Malaga, Pediatrics, Málaga, Spain. ${ }^{2}$ Hospital Materno Infantil de Malaga, Pediatric Infectology and Immunodeficiencies Unit, Málaga, Spain

3Compexo Hospitalario Universitario de Ourense, Pediatrics, Ourense, Spain. 4Regional Universitary Hospital of Málaga, Microbiology, Málaga, Spain.

\section{Background}

Clostridium difficile infection (CDI) has increased in children, although its diagnosis in $<3$ years-old is controversial due to high rates of colonization. Retrospective study to describe the characteristics of CDI diagnosed in children <16 years-old during 2013-2017,

$\mathrm{CDI}$ in $<1$ years-old were excluded following the recommendations of the literature.

\section{Case Presentation Summary}

Over the period, 30 episodes of CDI were diagnosed. 2 cases were excluded for being <1 years-old. The remaining 28 were diagnosed in 23 children. Mean age at diagnosis: 6.79 years (SD 4.68). 39.1 $\%$ were between 1-3 years-old. Risk factors (RF) for CDI are presented in figure 1.

Abdominal pain (57.9\%), diarrhea (46.3\%) and fever (10.5\%) were the predominant symptoms. $75 \%$ had mild-moderate colitis, no one presented as fulminant colitis. CD toxin was positive in $67.9 \%$ and Nucleic-Acid Amplification Test in $78.6 \%$; both resulted positive in $46.4 \%$.

Most of the first episodes were treated with metronidazole (68.4\%); rifaximine was used in $14.3 \%$ of them. The length of treatment was 10 days in $68 \%$. Two patients were not treated (7.1\%), with good outcome.

Six children developed recurrences (22.2\%), generally treated with metronidazole (33\%). One patient presented multiples recurrences and fidaxomicin therapy was indicated, without more relapses. The use of gastric acid-suppressing agents (GASA) or a previous episode of CDI was associated with the development of recurrence $(p<0.05$ and $p=0.06)$. No statistically association were observed between other RF and recurrence.

\section{Learning Points}

- Most CDI presented with mild-moderate colitis, as in other paediatric studies.

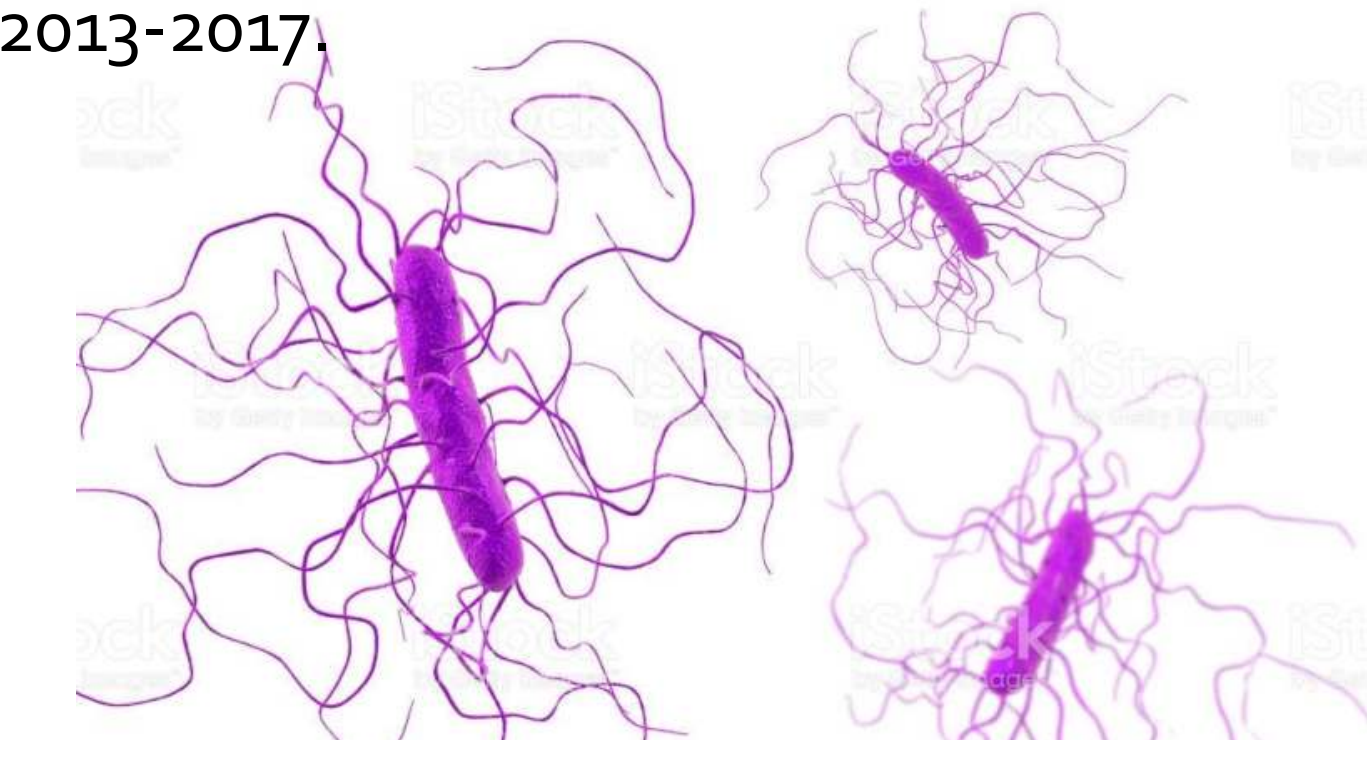

Risk Factors of CDI ( $n=28)$

\begin{tabular}{|c|c|}
\hline \multicolumn{2}{|c|}{ Risk Factors of CDI (n=28) } \\
\hline RF & $\mathbf{n ~ ( \% )}$ \\
\hline Immunosuppressive treatment & $14(50 \%)$ \\
\hline Previous hospitalization & $9(32,1 \%)$ \\
\hline Inflammatory bowel disease & $8(28,6 \%)$ \\
\hline Gastric acid-suppressing agents & $8(28,6 \%)$ \\
\hline Previous antibiotherapy & $8(28,6 \%)$ \\
\hline Previous CDI episode & $6(21,4 \%)$ \\
\hline Broad spectrum antibiotic therapy & $6(21,4 \%)$ \\
\hline Oncologic diseases & $5(17,9 \%)$ \\
\hline Figure 1. Risk factors of CDI. RF: Risk Factors; CDI: Clostridium difficile Infection
\end{tabular}

- 39.1\% CDI happened in <3 year-old children, although some of them could be simple colonization.

- Use of Gastric acid-suppressing agents was associated with relapsing CDI. 